\title{
Carrier phase bias estimation of geometry-free linear combination of GNSS signals for ionospheric TEC modeling
}

\author{
Anna Krypiak-Gregorczyk ${ }^{1}\left[\right.$ Pawel Wielgosz $^{1}$
}

Received: 3 September 2017 / Accepted: 6 February 2018 / Published online: 16 February 2018

(c) The Author(s) 2018. This article is an open access publication

\begin{abstract}
The ionosphere can be modeled and studied using multi-frequency GNSS signals and their geometry-free linear combination. Therefore, a number of GNSS-derived ionospheric models have been developed and applied in a broad range of applications. However, due to the complexity of estimating the carrier phase ambiguities, most of these models are based on low-accuracy carrier phase smoothed pseudorange data. This, in turn, critically limits their accuracy and applicability. Therefore, we present a new methodology of estimating the phase bias of the scaled L1 and L2 carrier phase difference which is a function of the ambiguities, the ionospheric delay, and hardware delays. This methodology is suitable for ionospheric modeling at regional and continental scales. In addition, we present its evaluation under varying ionospheric conditions. The test results show that the carrier phase bias of geometry-free linear combination can be estimated with a very high accuracy, which consequently allows for calculating ionospheric TEC with the uncertainty lower than 1.0 TECU. This high accuracy makes the resulting ionosphere model suitable for improving GNSS positioning for high-precision applications in geosciences.
\end{abstract}

Keywords GNSS $\cdot$ Carrier phase bias $\cdot$ Ionosphere $\cdot$ Ionospheric delay $\cdot$ TEC

\section{Introduction}

The ionospheric delay is one of the most dominant error sources in global navigation satellite systems (GNSS) positioning. Thus, reliable modeling of the ionospheric is one of the most challenging aspects of precise GNSS positioning (Leick et al. 2015; Wielgosz 2011) and GNSS-based geodetic and geodynamic studies (Bosy 2005). Currently, most of the available global, regional, and local ionosphere models are based on carrier phase smoothed pseudorange data, which presents low accuracy and requires strong smoothing to estimate the total electron content (TEC) (Brunini et al. 2004; Krypiak-Gregorczyk et al. 2013; Alizadeh et al. 2015). The ionospheric delay obtained from smoothed pseudoranges has an accuracy of several TEC units. One TECU $=10^{16} \mathrm{el} / \mathrm{m}^{2}$, and it is equivalent to $0.162 \mathrm{~m}$ of $\mathrm{L} 1$ signal delay. This is one of the reasons why most of the

Anna Krypiak-Gregorczyk

krypiakanna@wp.pl

Pawel Wielgosz

pawel.wielgosz@uwm.edu.pl

1 Institute of Geodesy, University of Warmia and Mazury in Olsztyn, Oczapowskiego 2, Olsztyn, Poland research centers use spherical harmonics expansion (SHE) or other heavily smoothing functions for TEC parameterization in their global and regional solutions (Schaer 1999; Hernández-Pajares et al. 2009; Schmidt et al. 2011). As a result, the obtained ionospheric maps are characterized by a low spatial resolution of several degrees and temporal resolution of 5-120 min. The widely used International GNSS Service (IGS) global model has a spatial resolution of $5.0^{\circ} \times 2.5^{\circ}$ and temporal resolution of $2 \mathrm{~h}$. The most recent studies show that the absolute TEC accuracy is rather low and amounts to 4-5 TECU (Hernandez-Pajares et al. 2017; Rovira-Garcia et al. 2016). Due to the very dynamic changes of the ionosphere, taking place not only during adverse solar activity events and magnetic storms, the accuracy and resolution offered by the current global and regional models are often not satisfactory.

The continuity of GNSS technology and its increasing precision make it one of the most efficient ways for TEC monitoring and modeling. On the other hand, the accurate knowledge of the state of the ionosphere plays a key role in determining a position using GNSS observations. This is due to the important influence of the ionospheric delays on determining carrier phase ambiguities. Therefore, the development of high-accuracy models with higher spatial 
and temporal resolution is required to support carrier phase ambiguity resolution (Wielgosz et al. 2005; Charoenkalunyuta and Satirapod 2014; Rovira-Garcia et al. 2016).

Achieving better accuracy than existing models may be possible through the use of the undifferenced carrier phase observations and their geometry-free linear combination to derive TEC estimates. Initial results are already provided in Krypiak-Gregorczyk et al. (2017), where we demonstrated that such a model was suitable to support ambiguity resolution in static relative positioning. Here, we focus on a new methodology that estimates the carrier phase bias of the geometry-free linear combination of GNSS-scaled carrier phase observations, i.e., the difference L1-L2, which is a function of the respective scaled ambiguities, the ionospheric delay, and hardware delays. This methodology is suitable for ionosphere modeling at regional and continental scales. Another goal is to validate the accuracy of the estimated phase bias of this function over a day-to-day boundary discontinuities.

\section{Methodology}

The data processing is based on a geometry-free linear combination $\left(L_{\mathrm{GF}}=\mathrm{L} 1-\mathrm{L} 2\right)$ of dual-frequency carrier phase GNSS observations. In general, when producing TEC maps, the overall processing is based on a three-step procedure:

Step 1 Estimation of the carrier phase bias.

Step 2 TEC calculation at the ionospheric piercing points (IPPs).

Step 3 TEC interpolation to form a regular grid (TEC map).

Therefore, the accuracy of the resulting TEC maps depends primarily on the accuracy of the estimated carrier phase bias for each continuous data arc. In other words, the accurate carrier phase bias is the prerequisite for any accurate TEC model. In this section, we provide basics on the ionosphere modeling together with our approach for solving the unknown carrier phase bias carried out in step (1).

\section{GNSS-TEC estimation}

The ionospheric effects on electromagnetic waves depend on the wave frequency. The ionosphere is a dispersive medium for GNSS signal frequencies, and this results in a delay of the ranging codes and an advance of the carrier phase. The ionospheric refraction is related to the signal frequency and the number of electrons on the ray path from a satellite to a receiver (Hofmann-Wellenhof et al. 2008). In the firstorder approximation, the ionospheric delay is inversely proportional to the square of its signal frequency (Hunsucker and Hargreaves 2002):

$\Delta I_{i}^{k}=\frac{40.3}{f^{2}} \mathrm{STEC}$

where $\Delta I_{i}^{k}$ is slant ionospheric delay (m), $k$ and $i$ are satellite and receiver indexes, $f$ is signal frequency $(\mathrm{Hz})$, and STEC is slant total electron content measured in units of electrons per meter squared $\left(\mathrm{el} / \mathrm{m}^{2}\right)$.

STEC is defined as the integrated electron density between the receiver and the satellite:

$\operatorname{STEC}_{i}^{k}=\int_{i}^{k} N_{e} \mathrm{~d} \rho$

where $N_{e}$ is electron density $\left(\mathrm{el} / \mathrm{m}^{3}\right), \rho$ is the physical distance along the given line of sight ( $\mathrm{LoS})$. The TEC depends on different factors such as location, time of day, season, solar, or geomagnetic activity. It is more generally measured in TECU $\left(1 \mathrm{TECU}=10^{16} \mathrm{el} / \mathrm{m}^{3}\right)$. The slant delay presented in (1) appears in the L1 and L2 carrier phase observables, which can be written as (in units of length):

$\mathrm{L}_{1}^{k}=q_{i}^{k}+c\left(\Delta t_{i}-\Delta t^{k}\right)+\Delta T_{i}^{k}-\Delta I_{i}^{k}-{ }_{1} \lambda \mathrm{N} 1_{i}^{k}+c\left(b_{\mathrm{L} 1}^{k}+b_{\mathrm{L} 1, i}\right)+\varepsilon$

$\mathrm{L} 2_{i}^{k}=q_{i}^{k}+c\left(\Delta t_{i}-\Delta t^{k}\right)+\Delta T_{i}^{k}-\xi \Delta I_{i}^{k}-{ }_{2} \lambda \mathrm{N} 2_{i}^{k}$ $+c\left(b_{\mathrm{L} 2}^{k}+b_{\mathrm{L} 2, i}\right)+\varepsilon$

where $q_{i}^{k}$ is geometric distance between satellite $k$ and receiver $i, \Delta t$ is clock error, $\Delta T_{i}^{k}$ is tropospheric delay, $\xi=\frac{f_{1}^{2}}{f_{2}^{2}}$ is a factor converting L1 ionospheric delay into L2 delay, $\lambda_{1}$ and $\lambda_{2}$ are signal wavelength, $\mathrm{N} 1$ and $\mathrm{N} 2$ are carrier phase ambiguities, $b_{\mathrm{L} 1}^{k}$ and $b_{\mathrm{L} 2}^{k}$ are satellite hardware delays, and $b_{\mathrm{L} 1, i}$ and $b_{\mathrm{L} 2, i}$ receiver hardware delays for both frequencies.

To extract information about the ionosphere from GNSS observations, a geometry-free linear combination $\left(L_{\mathrm{GF}}\right)$ of dual-frequency carrier phase observations is formed as:

$L_{i \mathrm{GF}}^{k}=\mathrm{L} 1_{i}^{k}-\mathrm{L} 2_{i}^{k}=-\xi_{\mathrm{GF}} \Delta I_{i}^{k}+B_{i \mathrm{GF}}^{k}$

where $L_{i \mathrm{GF}}^{k}$ is geometry-free linear combination, $\xi_{\mathrm{GF}}=1-\xi=1-\frac{f_{1}^{2}}{f_{2}^{2}} \approx-0.647$ is a factor that converts the ionospheric delay at $L_{\mathrm{GF}}$ signal to $\mathrm{L} 1, f_{1}$ and $f_{2}$ are $\mathrm{L} 1$ and L2 signal frequencies, $B_{i \mathrm{GF}}^{k}$ is carrier phase bias of the ionospheric function that combines ambiguity and hardware delays.

It should be noted that all frequency-independent effects such as receiver clock errors, satellites clock errors, and tropospheric delay are eliminated in (5). Unfortunately, this combination, in addition to information about the ionospheric delay, also includes carrier phase ambiguities and carrier phase hardware delays that have to be estimated 
(Böhm and Schuh, 2013). The carrier phase bias parameter $B_{\mathrm{iGF}}^{\mathrm{k}}$, combining the ambiguities and hardware delays, has an undefined wavelength. Therefore, it is expressed in units of length:

$B_{i \mathrm{GF}}^{k}=\lambda_{1} N_{i 1}^{k}-\lambda_{2} N_{i 2}^{k}-\left(b_{\mathrm{L} 1}^{k}-b_{\mathrm{L} 2}^{k}\right)-\left(b_{\mathrm{L} 1, i}-b_{\mathrm{L} 2, i}\right)$

where $b$ represents hardware delays (satellite and receiver), $N$ the carrier phase ambiguities, and $\lambda$ the signal wavelength. In this study, we made an attempt to accurately estimate the parameter $B_{\mathrm{iGF}}^{\mathrm{k}}$ through least squares adjustment of the relevant observational model, as described below.

\section{Estimation of the carrier phase bias}

In the presented approach, least squares is used for parameter estimation. A single carrier phase bias parameter $B_{\mathrm{iGF}}^{\mathrm{k}}$ lumping together the ambiguities and hardware delays is estimated for each continuous observation arc (6). In the data processing, the ionosphere $\left(\Delta I_{i}^{k}\right)$ can be parametrized every 10-20 min using a broad selection of different ionospheric parameterization such as spherical harmonics expansion (SHE), B-splines, general 2D polynomials, and local 2D polynomials (Krypiak-Gregorczyk et al. 2014). In our computations, we will be using SHE functions. The following parameterization is proposed:

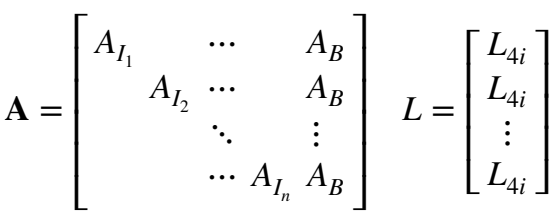

The design matrix $[A]$ consists of two groups of parameters: (1) epoch-dependent parameters $\left[A_{I}\right]$ representing the ionosphere and (2) constants $\left[A_{B}\right]$ representing the carrier phase biases for each continuous $L_{\mathrm{GF}}$ arc, while $L$ is the misclosures vector. We assume that all observables have the same precision and are uncorrelated; therefore, the weight matrix is the identity matrix. The system consists of a very large number of observations, and our internal tests showed that applying, e.g., elevation-dependent weighting scheme did not improve the results. Also note that due to spatial correlation of the ionosphere, the function parametrizing the ionosphere does need to have a high spatial resolution. Its errors average out over time when using a 24-h data set, and the resulting $B_{\mathrm{iGF}}^{\mathrm{k}}$ is estimated with a high accuracy as seen in subsequent sections. Note that the selected function is only used to estimate the carrier phase bias, and the function parameters are treated as nuisance ones. As mentioned above, the final TEC maps are obtained in step (3) and result from the interpolation of vertical TEC calculated in step (2), and the latter, in turn, is computed based on the estimated bias of step 1 . Note that any interpolation function may be used in step (3).

For the ionosphere modeling, a single layer model (SLM) with its associated mapping function is used (Schaer 1999; Shagimuratov et al. 2002). Due to the errors associated with a simple SLM mapping function, it is recommended to increase the elevation cutoff angle for GNSS observations to $20^{\circ}-30^{\circ}$.

\section{Regional ionosphere modeling at UWM in Olsztyn}

The proposed approach for estimating the carrier phase bias $B_{\mathrm{iGF}}^{\mathrm{k}}$ has already been used in the processing of a regional European ionospheric model developed at the University of Warmia and Mazury in Olsztyn (UWM). Its initial performance for Central Europe has been demonstrated in Krypiak-Gregorczyk et al. (2014). Our approach is based solely on precise undifferenced dual-frequency carrier phase data and the function $L_{\mathrm{GF}}$.

In the present study, observations from more than 200 GPS + GLONASS ground network stations were used. In particular, data from the EUREF Permanent Network (EPN) and European Position Determination System (EUPOS) stations were used to estimate the carrier phase bias (Bosy et al. 2007; Bruyninx et al. 2011). In the presented example, carrier phase biases for each continuous observation arcs were estimated together with parameters of the SHE functions. For the data preprocessing, which includes data cleaning and cycle slip detection, a 30-s sampling interval was used. The cycle slips detection problem is treated very carefully here, as any undetected slips affect the estimated carrier phase bias. Our detection approach is based on screening single and double $L_{\mathrm{GF}}$ time differences, similar to the method proposed by Cai et al. (2013). Any jump in double time differences over $\pm 0.1 \mathrm{~m}$ is treated as a cycle slip occurrence. In such a case, a new bias parameter is set up. Note that we do not try to repair any detected cycle slips. Also note, since the data comes from high-quality receivers mounted at the reference station networks, and the elevation cutoff angle is $20^{\circ}-30^{\circ}$, cycle slip occurrences are very rare in the processed data. Any undetected slips resulting in $L_{\mathrm{GF}}$ jumps under $\pm 0.1 \mathrm{~m}$ may affect the estimated bias. Our numerical tests based on actual GNSS data show that for some slip combination, e.g., -6 cycles on $\mathrm{L} 1$ and -5 cycles on $\mathrm{L} 2$, the effective jump on $L_{\mathrm{GF}}$ is about $8 \mathrm{~cm}$. This may pass undetected with the detection threshold of $\pm 0.1 \mathrm{~m}$. The data adjustment results show this $L_{\mathrm{GF}}$ slip of $8 \mathrm{~cm}$ causes error in the estimated bias up to $0.035 \mathrm{~m}$ and 0.3 TECU in the vertical TEC. On the other hand, our data analysis shows that double time differences of the $L_{\mathrm{GF}}$ are below $\pm 0.01 \mathrm{~m}$, and in case of slips the differences are usually over $\pm 1 \mathrm{~m}$. 
For instance, Zhao et al. (2015) reported a standard deviation of double time difference of the $L_{\mathrm{GF}}$ at the level of $4 \mathrm{~mm}$. Also, our method may be further improved when applying triple-frequency data to detect and repair cycle slips (Zhao et al. 2015). This is because repairing cycle slips allows for using longer data arcs for $B_{i \mathrm{GF}}^{k}$ estimation.

In this study, the data processing in step (1) was carried out with 1200-s sampling interval. Hence, 72 epochs were processed over a 24 -h period. The parameterization of the presented procedure makes the separation of the slant ionospheric delays and carrier phase biases in (5) possible. The biases are estimated in the step (1) of the regional ionospheric modeling. Then in the step (2), known carrier phase biases are substituted into (5) resulting in precise slant ionospheric delays at the IPPs. The slant ionospheric delays, in turn, are required for step (3) for subsequent calculation of the ionospheric vertical TEC. However, in this contribution we focus solely on the estimation and analysis of the carrier phase biases estimated in step (1).

\section{Accuracy analysis of carrier phase bias estimation}

The data from the EPN and EUPOS reference stations have been used to evaluate the performance of the proposed method for estimating the carrier phase biases. Dual-frequency carrier phase GPS and GLONASS observations from the $200+$ network stations are used in the numerical analysis to cover 7 days during March 7-13, 2012 (DOY 67-73). The selected period was characterized by varying ionosphere conditions, including a strong geomagnetic storm. Figure 1 depicts the disturbance storm time index (Dst). As one can see in the figure, the test period covers 2 days before the storm (DOY 67-68) and also the 4 days (DOY 70-73) after the main storm (DOY 69) that was characterized by DST dropping down to $-145 \mathrm{nT}$.

In order to investigate the accuracy of the carrier phase bias estimation, we validated our method by analyzing of the bias differences at the day-to-day boundaries for adjacent

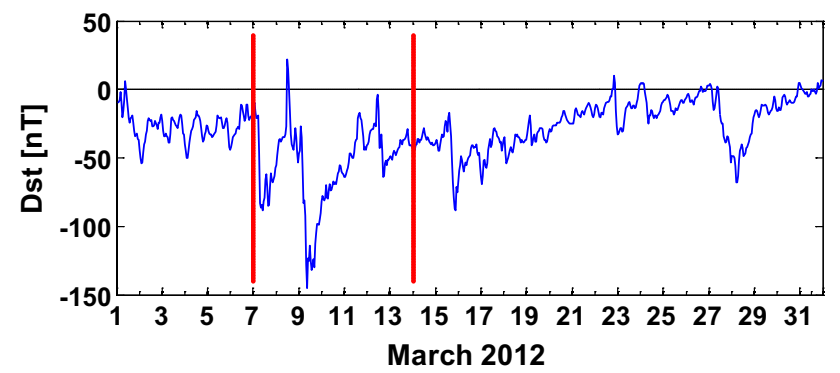

Fig. 1 Variations of the disturbance storm time index (Dst) during March 2012 (red bars limit test period) 24-h arcs. Note that the $L_{\mathrm{GF}}$ carrier phase bias is estimated using 24-h datasets. A similar approach is used, e.g., to validate the accuracy of precise orbits of GNSS satellites (Griffiths and Ray 2009; Tegedor et al. 2014). Moreover, we also present the analysis of TEC differences calculated at adjacent epochs at 23:59:30 on the first day and at 0:00:00 on the second day of a particular satellite arc. Note that TEC at both epochs comes from different and independent bias estimates that are computed from different daily RINEX files. This is also the case for the bias comparisons at dayto-day boundaries.

For the statistical analysis of the estimated carrier phase biases, 12 references stations from the EPN network located in Central Europe were selected (Fig. 2). The differences between the carrier phase bias values over day boundaries were analyzed as a metric of their accuracy. The processing was carried out in two variants. In order to reduce errors associated with the SLM mapping function, the elevation cutoff angle for GNSS observations was set to $30^{\circ}$ in the first variant and to $20^{\circ}$ in the second variant. This allowed for analyzing the impact of the selected elevation cutoff angle. Note that decreasing the cutoff angle increases the number of available observations, but on the other hand, low-elevation data may be biased by the inaccuracy of the SLM. Note, however, that when using vertical TEC from ionospheric maps to correct low-elevation data at $10^{\circ}-15^{\circ}$, one also may expect larger errors. Therefore, for future application over Europe we recommend using the recently developed Barcelona Ionospheric Mapping function, which performs better at low elevations (Lyu et al. 2018).

Figure 2 illustrates locations of all test stations, and Fig. 3 illustrates examples of the location of the all analyzed arcs for the test stations. Arc segments from different days are marked with different colors. The red lines refer to the end

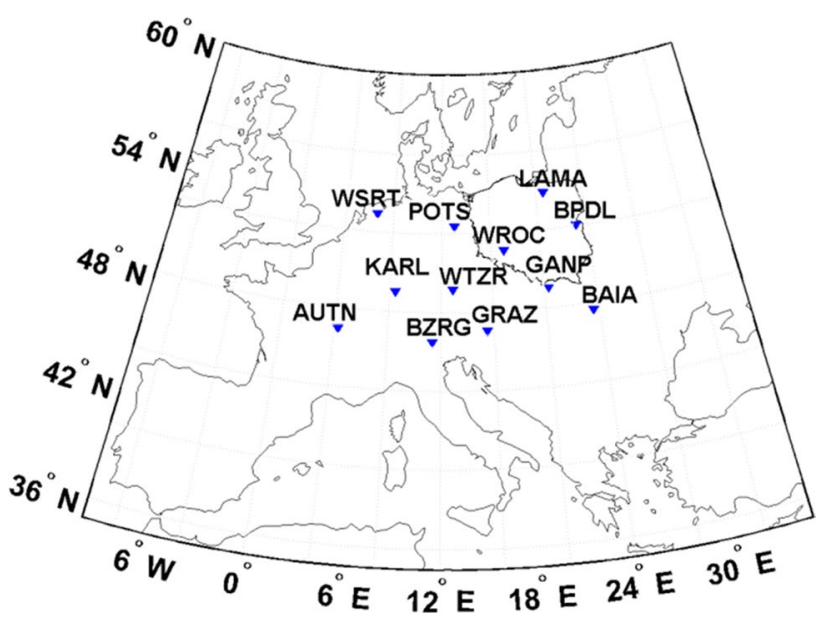

Fig. 2 Locations of EPN station used in the statistical analysis 


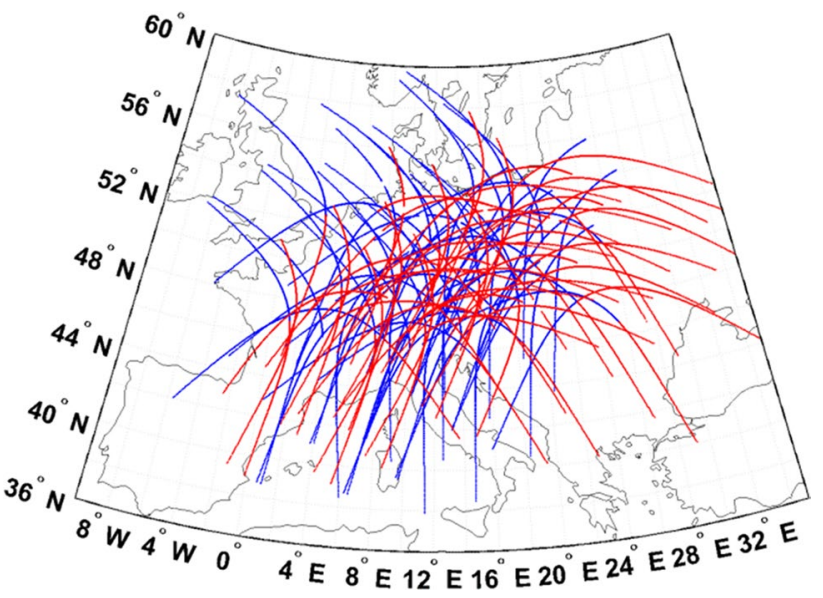

Fig. 3 Location of the all analyzed GPS and GLONASS data arcs over day boundary between DOY 69 (blue) and 70 (red) for all selected stations of the first day, and the blue lines refer to the beginning of the next day.

Day boundary discontinuities of the estimated $L_{\mathrm{GF}}$ biases $B_{i \mathrm{GF}}^{k}$ were calculated for each available data arc and each test station. Then RMS of bias differences for each satellite and station pair over the project time were calculated. In addition, for graphical representation, example daily bias discontinuities were calculated and are presented in Figs. 4 and 5 for four selected test stations LAMA, WROC, AUTN, and WSRT and three GPS satellites. Note that we processed data from all available GPS and GLONASS satellites, but for figure clarity we present representative results for several selected cases. Full results for all satellites and stations are provided in Tables 1 and 2. Blue, green and yellow bars in the figures correspond to the differences between the carrier phase bias values over day boundaries for PRN25, PRN29, and PRN31, respectively. Red lines denote bias difference values that correspond to 1 and 2 TECU levels, as related to $L_{\mathrm{GF}}$ signal.

In general, the bias differences for all four stations reach the lowest values during active days and then increase during
Fig. 4 Example $B_{i \mathrm{GF}}^{k}$ differences over day boundaries for PRN 25, 29, and 31 and LAMA, WROC, AUTN, and WSRT stations, results for 30-degree elevation mask
Fig. 5 Example $B_{i \mathrm{GF}}^{k}$ differences over day boundaries for PRN 25, 29, and 31 and LAMA, WROC, AUTN, and WSRT stations, results for 20-degree elevation mask
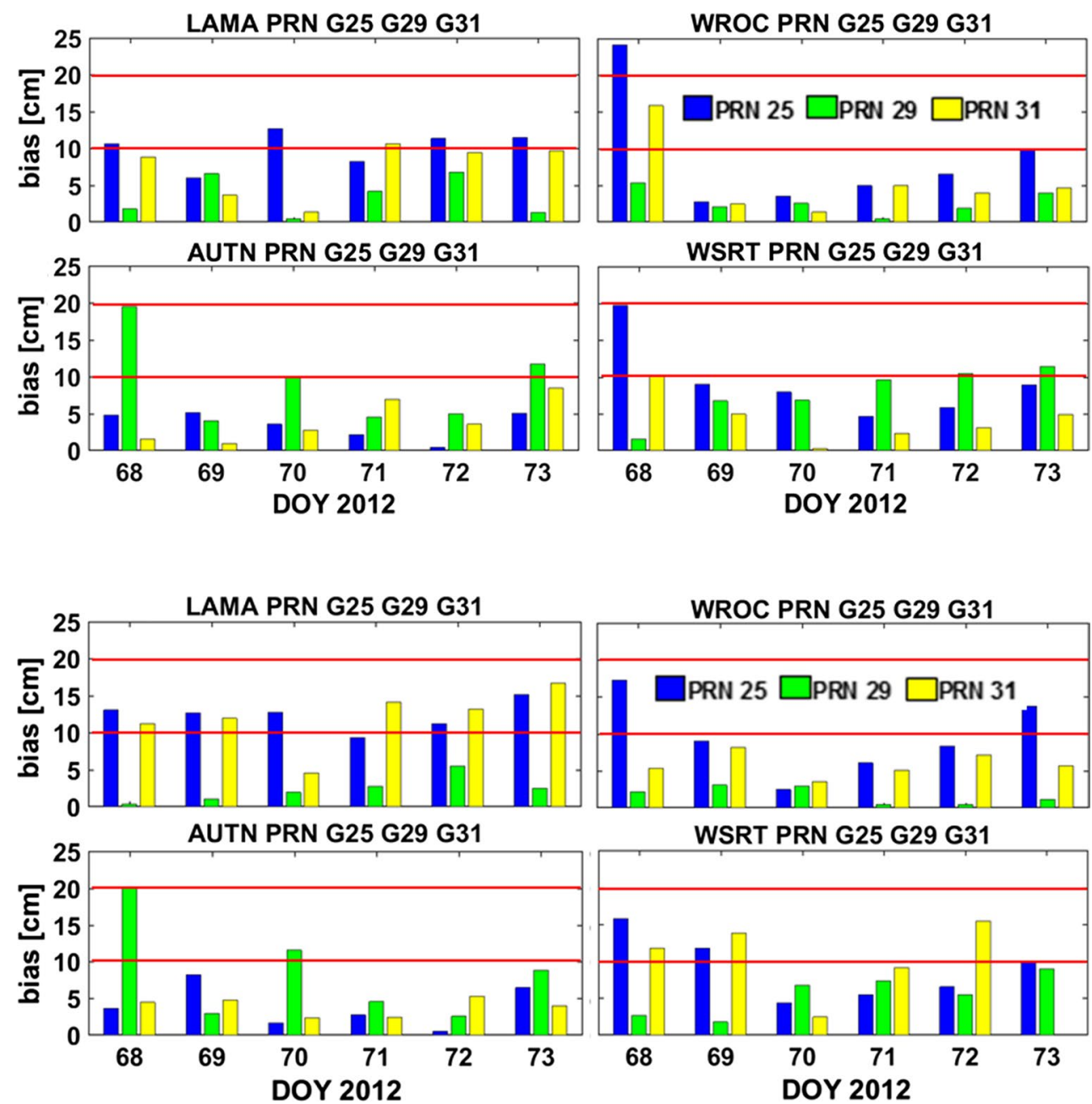
Table 1 Statistics of the carrier phase bias differences over day boundary discontinuities (30-degree elevation mask) (m)
Table 2 Statistics of the carrier phase bias differences over day boundary discontinuities (20-degree elevation mask) (m)

\begin{tabular}{lllllll}
\hline DOY & RMS & Min & Max & Mean & $< \pm 10 \mathrm{~cm}(\%)$ & $< \pm 20 \mathrm{~cm}(\%)$ \\
\hline $67 / 68$ & 0.09 & -0.20 & 0.20 & 0.03 & 75.00 & 100.00 \\
$68 / 69$ & 0.07 & -0.11 & 0.15 & 0.03 & 89.47 & 100.00 \\
$69 / 70$ & 0.06 & -0.12 & 0.13 & 0.03 & 91.23 & 100.00 \\
$70 / 71$ & 0.05 & -0.10 & 0.13 & 0.02 & 95.92 & 100.00 \\
$71 / 72$ & 0.07 & -0.14 & 0.15 & 0.02 & 79.25 & 100.00 \\
$72 / 73$ & 0.07 & -0.12 & 0.14 & 0.02 & 78.57 & 100.00 \\
Mean & 0.07 & & & & 84.91 & 100.00
\end{tabular}

\begin{tabular}{llllllc}
\hline DOY & RMS & Min & Max & Mean & $< \pm 10 \mathrm{~cm}(\%)$ & $< \pm 20 \mathrm{~cm}(\%)$ \\
\hline $67 / 68$ & 0.10 & -0.20 & 0.27 & 0.04 & 70.77 & 92.31 \\
$68 / 69$ & 0.09 & -0.05 & 0.21 & 0.06 & 66.67 & 98.48 \\
$69 / 70$ & 0.06 & -0.12 & 0.13 & 0.02 & 92.86 & 100.00 \\
$70 / 71$ & 0.06 & -0.12 & 0.14 & 0.02 & 90.00 & 100.00 \\
$71 / 72$ & 0.07 & -0.15 & 0.18 & 0.03 & 78.57 & 100.00 \\
$72 / 73$ & 0.10 & -0.28 & 0.19 & 0.01 & 71.25 & 93.75 \\
Mean & 0.08 & & & & 78.35 & 97.42 \\
\hline
\end{tabular}

the first and the last days of the test period. In the presented examples, the residuals for station WROC, located in the center of the model area, are clearly lower than those for LAMA and WSRT, and most of them are within 5-10 cm range for the first variant, and about $10 \mathrm{~cm}$ for the second variant, except for PRN25, showing residuals of about $20 \mathrm{~cm}$ for $20^{\circ}$ cutoff. Station AUTN located in the west achieved slightly worse results than station WROC, but clearly better than LAMA and WSRT. As one can see in the figures, in all cases the biases estimated using observations with the 30-degree elevation cutoff present slightly lower differences compared to the biases estimated using a $20^{\circ}$ cutoff. In both variants, for stations LAMA, WROC, and WSRT the bias differences over day boundaries present the highest values for PRN25. The fourth station, station AUTN, achieved almost the lowest values for PRN25 while the results for PRN29 reached $20 \mathrm{~cm}$ for both variants. It is observed from the figures that the differences for LAMA and WSRT are characterized by the highest values reaching $12 \mathrm{~cm}$ for 30 -degree elevation mask and $15 \mathrm{~cm}$ for the $20^{\circ}$ mask. Since stations LAMA and WSRT are located in the northern part of our model, the larger differences of the bias over day boundaries for LAMA and WSRT are caused by fewer observations from this region used for the bias estimation.

Statistics concerning mean differences between the bias values over day boundaries, their minimum, maximum and the overall RMS for the two analyzed elevation cutoff angles and all 7 days of the test period and all GPS and GLONASS satellites are presented in Tables 1 and 2. In the tables, the percentiles of the bias differences falling within arbitrarily selected limits of $\pm 10 \mathrm{~cm}$ and $\pm 20 \mathrm{~cm}$ are presented. These limits correspond to 1.0 and 2.0 TECU. It should be noted that we did not notice effects depending on satellite block or constellation.

The results in Table 1 show that during all analyzed days the RMS does not exceed $10 \mathrm{~cm}$. In the first variant $\left(30^{\circ}\right)$, the RMS values amounts to $9 \mathrm{~cm}$ at the beginning of the test period, but at the end fall to $7 \mathrm{~cm}$ (Table 1). For active days 68/69 and 69/70 the RMS reached only $5 \mathrm{~cm}$, and it was similarly low over the next boundary (70/71). The mean absolute differences did not exceed $5 \mathrm{~cm}$ and for active day boundary 68/69; these values reached only $3 \mathrm{~cm}$. In addition, the analyses show that the application of the presented approach results in the carrier phase bias differences in the $\pm 20 \mathrm{~cm}$ range in $100 \%$ cases for the first variant. It should be noted that for the whole analyzed period more than $84 \%$ of differences are within $\pm 10 \mathrm{~cm}$ range.

In the second variant, as it might be expected, the accuracy of the carrier phase bias estimation decreased (Table 2). However, the RMS values increased by $1 \mathrm{~cm}$ only compared to the first variant. A significant difference in the results from the two variants is evident in case of differences falling within $\pm 10 \mathrm{~cm}$ and $\pm 20 \mathrm{~cm}$ ranges. This metric confirms the lower accuracy of the bias estimation in case of the second variant. It should be noted that increasing the elevation cutoff (variant 1) improves the resulting bias accuracy. However, this reduces the number of usable observations from 1898 IPPs to 1717 IPPs during a single day, which is about $10 \%$. This in turn may affect the accuracy of the derived vertical TEC maps.

It can be seen that on the stormy day the accuracy of the $L_{\mathrm{GF}}$ carrier phase bias estimation is significantly higher as 
Table 3 Daily mean standard deviations $\sigma_{x}$ in meters of the estimated $B_{i \mathrm{GF}}^{k}$ for 20 - and 30-degree elevation cutoff

\begin{tabular}{lll}
\hline DOY & $\sigma_{x\left(20^{\circ}\right)}$ & $\sigma_{x\left(30^{\circ}\right)}$ \\
\hline 67 & 0.06 & 0.06 \\
68 & 0.06 & 0.05 \\
69 & 0.06 & 0.06 \\
70 & 0.05 & 0.05 \\
71 & 0.04 & 0.05 \\
72 & 0.04 & 0.04 \\
73 & 0.04 & 0.04 \\
Mean & 0.05 & 0.05 \\
\hline
\end{tabular}

compared to the quiet days. It should be noted, however, that the main phase of this storm was characterized by a decrease of the electron content. That may explain the higher bias estimation accuracy during the storm.

In addition, we evaluated the obtained RMS against the standard deviation $\sigma_{x}$ derived from the variance-covariance matrix resulting from the adjustment (Table 3). In general, the daily mean $\mathrm{m}_{\mathrm{x}}$ is $30-40 \%$ lower than the daily RMS. This may suggest that the formal error is slightly underestimated. Also, the difference in $\mathrm{m}_{\mathrm{x}}$ derived with different elevation cutoff angles is less pronounced.

The high accuracy of the estimated biases is also reflected in the accuracy of the resulting vertical TEC values. The estimated carrier phase biases were used to derive precise slant ionospheric delays at the IPPs. Following (5),

$\Delta I_{i}^{k}=\frac{L_{i \mathrm{GF}}^{k}-B_{i \mathrm{GF}}^{k}}{-\xi_{4}}$
The slant delays were subsequently converted to the vertical delays using a SLM mapping function. This procedure is routinely executed in step (2) of our model. Tables 4 and 5 represent statistics of the vertical TEC differences over day boundaries for the two variants of 30- and 20-degree elevation mask, respectively. In particular, the TEC was calculated using the last epoch from the first day and the first epoch from the next day; however, note the $30 \mathrm{~s}$ of the time difference. The resulting RMS did not exceed 0.80 TECU for both cutoff variants, with mean values of 0.55 TECU and 0.62 TECU for the first and the second variants, respectively. Most of the vertical TEC differences are within the \pm 1 TECU limit, namely 94 and $87 \%$, for the first and second variants, respectively.

\section{Conclusions}

We presented and validated a methodology for accurate bias estimation of the phase function $L_{\mathrm{GF}}$ for use in GNSSbased regional ionospheric modeling. The methodology allows for subsequent TEC calculation using exclusively high-accuracy carrier phase data. The accuracy of the estimated bias was determined by analysis of its repeatability over the day-to-day boundaries for GPS and GLONASS satellites. Test results show that the bias accuracy (RMS) is at the level of 7-8 cm, i.e., below 1 TECU depending on the selected data elevation cutoff. Note that the RMS may be partially impacted by undetected small cycle slip combinations. The RMS is slightly higher than the formal
Table 4 Statistics of the vertical TEC differences over day boundary discontinuities (30-degree elevation mask) (TECU)

Table 5 Statistics of the vertical TEC differences over day boundary discontinuities (20-degree elevation mask) [TECU]

\begin{tabular}{lllllll}
\hline DOY & RMS & Min & Max & Mean & $<0.5$ TECU $(\%)$ & $<1$ TECU $(\%)$ \\
\hline $67 / 68$ & 0.72 & 0.02 & 1.81 & 0.55 & 56.14 & 87.72 \\
$68 / 69$ & 0.54 & 0.03 & 1.33 & 0.46 & 61.40 & 96.49 \\
$69 / 70$ & 0.49 & 0.00 & 1.11 & 0.39 & 68.97 & 96.55 \\
$70 / 71$ & 0.42 & 0.00 & 1.07 & 0.34 & 76.00 & 98.00 \\
$71 / 72$ & 0.57 & 0.02 & 1.21 & 0.48 & 60.38 & 94.34 \\
$72 / 73$ & 0.58 & 0.00 & 1.11 & 0.47 & 61.40 & 89.47 \\
Mean & 0.55 & & & & 64.05 & 93.76 \\
\hline
\end{tabular}

\begin{tabular}{lllllll}
\hline DOY & RMS & Min & Max & Mean & $<0.5$ TECU $(\%)$ & $<1$ TECU $(\%)$ \\
\hline $67 / 68$ & 0.79 & 0.00 & 2.08 & 0.59 & 61.54 & 76.92 \\
$68 / 69$ & 0.74 & 0.02 & 1.79 & 0.61 & 46.15 & 81.54 \\
$69 / 70$ & 0.46 & 0.02 & 1.13 & 0.38 & 71.43 & 97.14 \\
$70 / 71$ & 0.45 & 0.00 & 1.13 & 0.36 & 76.27 & 94.92 \\
$71 / 72$ & 0.57 & 0.01 & 1.51 & 0.45 & 59.70 & 91.04 \\
$72 / 73$ & 0.69 & 0.01 & 1.58 & 0.52 & 61.84 & 81.58 \\
Mean & 0.62 & & & & 62.82 & 87.19 \\
\hline
\end{tabular}


standard deviations derived from the variance matrix, which amounted to $4-6 \mathrm{~cm}$.

In our opinion, these results confirm the high accuracy of our method that may be applicable to computing accurate ionospheric models. Such models may be used, e.g., to analyze disturbed states of the ionosphere or to support GNSS data processing, both absolute and relative, in high-accuracy applications in geosciences.

Acknowledgements The research is supported by Grant No. UMO2013/11/B/ST10/04709 from the Polish National Center of Science.

Open Access This article is distributed under the terms of the Creative Commons Attribution 4.0 International License (http://creativecommons.org/licenses/by/4.0/), which permits unrestricted use, distribution, and reproduction in any medium, provided you give appropriate credit to the original author(s) and the source, provide a link to the Creative Commons license, and indicate if changes were made.

\section{References}

Alizadeh MM, Schuh H, Schmidt M (2015) Ray tracing technique for global 3-D modeling of ionospheric electron density using GNSS measurements. Radio Sci 50(6):539-553

Böhm J, Schuh H (2013) Atmospheric effects in space geodesy. Springer, Berlin. https://doi.org/10.1007/978-3-642-36932-2

Bosy J (2005) Data processing of local GPS networks located in a mountain area. Acta Geodyn Geomater 3(139):49-56

Bosy J, Graszka W, Leonczyk M (2007) ASG-EUPOS-a multifunctional precise satellite positioning system in Poland. Eur J Nav 5:2-6

Brunini C, Meza A, Azpilicueta F, Zele MAV (2004) A new ionosphere monitoring technology based on GPS. Astrophys Space Sci 290(3-4):415-429

Bruyninx C, Baire Q, Legrand, Roosbeek F (2011) The EUREF permanent network (EPN): recent developments and key issues. Presented at EUREF 2011 symposium, Chisinau, 25-28 May 2011

Cai C, Liu Z, Xia P, Dai W (2013) Cycle slip detection and repair for undifferenced GPS observations under high ionospheric activity. GPS Solut 17(2):247-260

Charoenkalunyuta T, Satirapod C (2014) Effect of Thai Ionospheric Maps (THIM) model on the performance of network based RTK GPS in Thailand. Surv Rev 46(334):1-6

Griffiths J, Ray JR (2009) On the precision and accuracy of IGS orbits. J Geod 83(3-4):277-287. https://doi.org/10.1007/s00190-008-0237-6

Hernandez-Pajares M, Roma-Dollase D, Krankowski A, Garcia-Rigo A, Orús-Perez R (2017) Methodology and consistency of slant and vertical assessments for ionospheric electron content models. J Geodesy 91(12):1-10. https://doi.org/10.1007/s00190-017-1032-z

Hernández-Pajares M, Juan JM, Sanz J, Orus R, Garcia-Rigo A, Feltens J, Komjathy A, Schaer S, Krankowski A (2009) The IGS VTEC maps: a reliable source of ionospheric information since 1998. J Geod 83(3-4):263-275. https://doi.org/10.1007/s00190-008-0266-1
Hofmann-Wellenhof B, Lichtenegger H, Wasle E (2008) GNSS global navigation satellite systems; GPS, GLONASS, Galileo \& more. Springer, New York

Hunsucker RD, Hargreaves JK (2002) The high-latitude ionosphere and its effects on radio propagation. Cambridge University Press, Cambridge

Krypiak-Gregorczyk A, Wielgosz P, Gosciewski D, Paziewski J (2013) Validation of approximation techniques for local total electron content mapping. Acta Geodyn Geomater 10(3):275-283. https://doi. org/10.13168/AGG.2013.0027

Krypiak-Gregorczyk A, Wielgosz P, Krukowska M (2014) A new ionosphere monitoring service over the ASG-EUPOS network stations. In: 9th international conference environmental engineering (9TH ICEE)—selected papers. https://doi.org/10.3846/enviro.2014.224, http://leidykla.vgtu.lt/conferences/ENVIRO_2014/Articles/5/224_ Krypiak_Gregorczyk.pdf

Krypiak-Gregorczyk A, Wielgosz P, Jarmołowski W (2017) A new TEC interpolation method based on the least squares collocation for high accuracy regional ionospheric maps. Meas Sci Technol 28(4):045801. https://doi.org/10.1088/1361-6501/aa58ae

Leick A, Rapoport L, Tatarnikov D (2015) GPS satellite surveying, 4th edn. Wiley, Hoboken

Lyu H, Hernández-Pajares M, Nohutcu M, García-Rigo A, Zhang H, Liu J (2018) The Barcelona Ionospheric Mapping Function (BIMF) and its application to northern mid-latitudes. GPS Sol (accepted)

Rovira-Garcia A, Juan JM, Sanz J, González-Casado G, Ibáñez D (2016) Accuracy of ionospheric models used in GNSS and SBAS: methodology and analysis. J Geod 90(3):229-240

Schaer S (1999) Mapping and Predicting the Earth's Ionosphere Using the Global Positioning System. Ph.D. dissertation Astronomical Institute University of Berne

Schmidt M, Dettmering D, Moßmer M, Wang Y, Zhang J (2011) Comparison of spherical harmonic and B spline models for the vertical total electron content. Radio Sci 46(6):RS0D11. https://doi. org/10.1029/2010RS004609

Shagimuratov I, Baran LW, Wielgosz P, Yakimova GA (2002) The structure of mid- and high-latitude ionosphere during September 1999 storm event obtained from GPS observations. Ann Geophys 20(6):665-671

Tegedor J, Ovstedal O, Vigen E (2014) Precise orbit determination and point positioning using GPS, Glonass, Galileo and BeiDou. J Geod Sci 4(1):65-73. https://doi.org/10.2478/jogs-2014-0008

Wielgosz P (2011) Quality assessment of GPS rapid static positioning with weighted ionospheric parameters in generalized least squares. GPS Solut 15(2):89-99. https://doi.org/10.1007/s10291-010-0168-6

Wielgosz P, Kashani I, Grejner-Brzezinska DA (2005) Analysis of long-range network RTK during severe ionospheric storm. J Geod 79(9):524-531. https://doi.org/10.1007/s00190-005-y

Zhao Q, Sun B, Dai Z, Hu Z, Shi Ch, Liu J (2015) Real-time detection and repair of cycle slips in triple-frequency GNSS measurements. GPS Solut 19:381. https://doi.org/10.1007/s10291-014-0396-2 


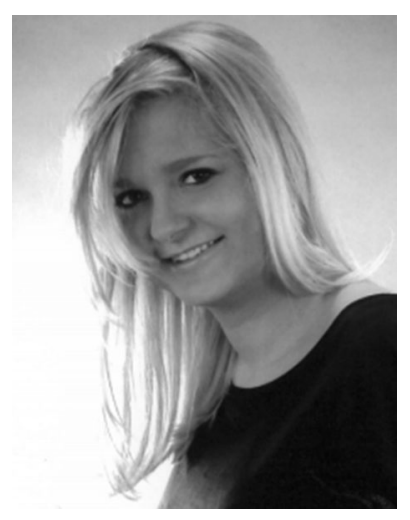

Anna Krypiak-Gregorczyk is an Assistant Professor at the Institute of Geodesy of the University of Warmia and Mazury in Olsztyn (UWM). Her research interests cover GNSS-based ionosphere study. She is a member of WG4.3.1 "Real-time Ionosphere Monitoring" within the International Association of Geodesy (IAG).

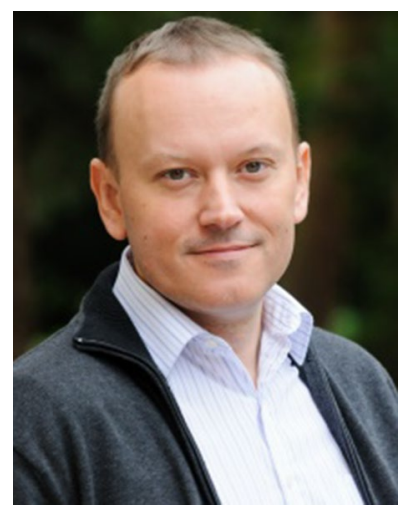

Pawel Wielgosz is an Associate Professor at the Faculty of Geodesy, Geospatial and Civil Engineering of the University of Warmia and Mazury in Olsztyn (UWM), Poland. His research interests cover satellite navigation, precise kinematic and static positioning, deformation monitoring, and also GNSS-based ionosphere and troposphere study. He is the chair of the International Association of Geodesy (IAG) Sub-Commission 4.4 "Multi-constellation GNSS." 